\title{
Automated screening of cervical smears using immunocytochemical staining: a possible approach
}

\author{
BILYANA VALKOVA, DJR LAURENCE \\ From the Ludwig Institute for Cancer Research (London Branch), Royal Marsden Hospital, Sutton, Surrey
}

SUMMARY A preliminary analysis was undertaken to ascertain whether a Leitz TAS (Textur Analyse System) video camera system, which is computer controlled, could be used to detect abnormal cells in cervical smears. Immunocytochemical together with haemalum nuclear staining was used for the epithelial membrane antigen. It was possible to detect abnormal cells in routinely prepared smears taken from patients with various degrees of cervical neoplasia, including the earliest stages, but metaplastic cells were not detected by the system. Smears from patients assessed as normal by routine cytology gave a low incidence of positive results due to occasional overlapping of cells or the presence of air bubbles. This system seems to offer a new possibility for rapid automated cytometry of cervical smears.

The widespread use of manual screening for the early diagnosis of cervical cancer and the high cost of this screening have prompted research into automated systems. For automatic discrimination between normal, premalignant, and malignant cells several cell variables have been used: cell diameter; nuclear : cytoplasmic ratio; nuclear density; and the so called Minkowski gradient, which relates area, perimeter, and connectivity of the cell image..$^{1-7}$ The programmes designed to evaluate these variables require from three to six minutes for each slide. ${ }^{7-9}$ Erhardt et al reported that parallel processing should enable a high resolution analysis of a sample containing several thousand cells to be obtained in about one minute.' The principal tasks of a high resolution system for automated cytology are locating the cell, segmentation, and feature extraction of the cell image, as described by Erhardt et al.'

The aim of this study was to try a new approach to the speedy but reliable detection of malignant and premalignant cells in cervical smears, using immunocytochemical staining with monoclonal or polyclonal antibodies, or both for the epithelial membrane antigen, and haemalum nuclear counter staining. ${ }^{10}$ This is a standard histochemical procedure, but further details of the application to epithelial membrane antigen immunocytochemistry have been reported elsewhere. ${ }^{1012}$

\section{Material and methods}

Cervical cells were obtained, using a wooden Ayres Accepted for publication 3 May 1985 spatula or an acryl cotton tipped applicator, from 37 patients attending family planning and gynaecological clinics in the London area. Two specimens were taken from each woman. The first was used for conventional Papanicolaou staining, the second for immunocytochemical staining. The specimens for immunocytochemistry were stored before evaluation in Cellfix $(0.1 \%$ dithiothreitol, $0.05 \mathrm{M}$ phosphate buffer $\mathrm{pH} 7.0$ in $40 \% \mathrm{v} / \mathrm{v}$ aqueous ethanol) as recommended by Husain et al. ${ }^{7}$ The cells were dislodged by agitation and then dispersed by syringing. ${ }^{10}$ They were centrifuged, resuspended, and washed twice in phosphate buffered saline, $\mathrm{pH} \mathrm{7.2}$ (the final cell suspension used to form the smear was a 1:2 dilution based on the volume of the final cell pellet). The preparations for the monolayer were made as described by Mukawa et al. ${ }^{9}$ Two $25 \mu \mathrm{l}$ drops of the cell suspension were placed on a slide and spread to an area of $800 \mathrm{~mm}^{2}$. The suspensions were spread by shearing between parallel slides. ${ }^{9}$ This was a helpful technique in eliminating overlapping clumps of cells that could have given false signals in the subsequent analysis. These preparations were consistent with the special requirements of the Leitz TAS automated analysis of the image when applied to automated screening of smears, as described by Schwarz et al."

The preparations were stained by an indirect immunoalkaline phosphatase method using either polyclonal rabbit antisera or two monoclonal antibodies (77.1 and 8.30.3). ${ }^{10} 12$ Alkaline phosphatase was shown by the formation of an azodye between naphthol AS BI base (CI 37085) released by action 
of the enzyme and fast red TR salt (CI 37566), a standard histochemical procedure. ${ }^{13}$

In our study the binding of the antiepithelial membrane antigen and second antibody and the subsequent enzymatic reaction were each carried out during incubation for one hour at room temperature, which caused the cytoplasm and the membrane of abnormal cells to be stained deep red. The nuclei were counterstained blue with Mayer's haemalum for five minutes followed by washing in

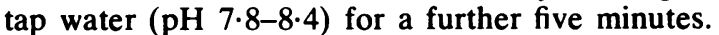
The haemalum stain was made by the iodate oxidation method. ${ }^{14}$ The development of the blue colour was accelerated by a one to two second exposure of the stained slides to saturated lithium carbonate solution. The immunocytochemically stained smears were mounted in glycerol jelly ( $\mathrm{pH} 5.8)^{14}$ and evaluated by automated screening. Papanicolaou stained smears were evaluated by the cytology department servicing the originating clinic. Image analysis of the immunocytochemically stained cells was performed with a Leitz TAS apparatus, comprising a microscope and a video camera together with computer

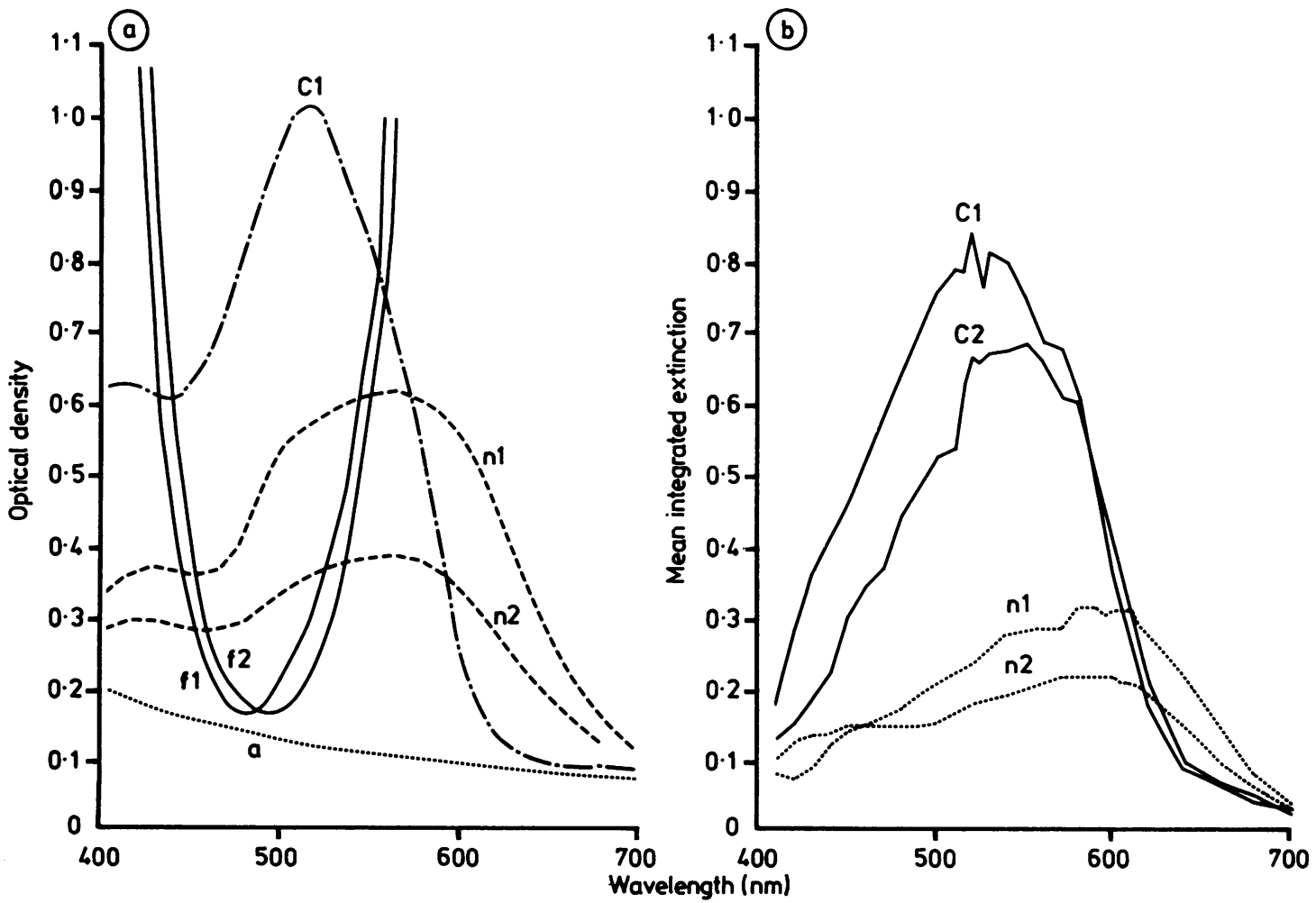

Fig. 1 Absorption spectra of antiepithelial membrane antigen azodye product and haemalum stain (a) deposited within transparent foils and (b) by microdensitometry in suitable cells. Curves labelled c1 and c2 are spectra of red azodye that stains cytoplasm of epithelial membrane antigen positive cells. Curves $n 1$ and $n 2$ are haemalum nuclear stain. Curve a is scattering background from unstained foil.

control and data analysis. This system has been used to study cervical smears according to nuclear size and density. ${ }^{15}$

The optical system of TAS includes a $100 \mathrm{~W} 12 \mathrm{~V}$ quartz halogen lamp with a tungsten filament run at a colour temperature of $3400^{\circ} \mathrm{K}$. There are two infrared absorbing filters in the lamp housing. The light passing through the specimen is focused by the microscope on to the photosensitive surface of a 0.25 inch Plumbicon video camera tube (peak response at $500 \mathrm{~nm}$ ). Between the lamp and the microscope an Ilford 307 "cyan" gelatine filter was inserted (maximum transmission at $480 \mathrm{~nm}$ in white light). Correction for the red bias of the light source and for the wavelength response of the video camera shifted the maximum effective transmission to 500 nm (Fig. 1a curves $\mathrm{f} 1$ and $\mathrm{f} 2$ ).

Fig. 1 shows the absorption of the azodye used to detect epithelial membrane antigen in the cytoplasm and of the haemalum nuclear stain. These spectra were obtained by macroscopic evaluation of stained foils and microscopic evaluation using a scanning and integrating microdensitometer for stained cervi- 
cal smears (Vickers type M85a). The stained foils (Fig. 1a) were prepared as described by Baker ${ }^{15}$ except that Visking cellulose acetate sheet cut into 3 $\times 1 \mathrm{~cm}$ pieces from dialysis tubing was used rather than formolised gelatine.

Napthol AS BI base was put on the sheet in 50\% aqueous dimethylformamide, and, after excess solution had been wiped off, the ayodye was developed in a solution of fast TR salt and sodium bicarbonate. Other sheets were impregnated with haemalum solution in a similar manner, and the dye was fixed by placing the foil in a dilute bicarbonate solution after an initial rinse in tap water.

Microscopic evaluation (Fig. 1b) entailed measuring the spectrum of the cytoplasm of two cells strongly stained with the red azo dye and the nuclei of two other cells with weak cytoplasmic staining. In both Figs. 1a and $1 \mathrm{~b}$ the maximum absorption of the red staining is at $510 \mathrm{~nm}$ and that of the blue nuclear staining at $570 \mathrm{~nm}$. The spectrum of the nuclear staining agrees with that described by Baker. ${ }^{15}$

Computer analysis of the image produced by the video camera was carried out using the TAS system. The output from the video camera was digitised into a $256 \times 256$ set of picture elements (pixels) arranged in a hexagonal network. Two main controls were exerted in converting this output into an image for computer memory-namely, control of thresholds and erosion of the initially formed image.

The threshold controls consist of two console settings, one of which was kept at zero while the other was initially varied by equal steps until an optimal value was obtained (Fig. 2). The threshold controls thereby determine the minimum optical density befow which the computer will not register the presence of a discernible feature in the microscopic field. Each pixel is mapped on to a single element (bit) of computer memory. The total array of elements, related logically to the spatial array of pixels and hence to the arrangement of the microscopic field, is known as a "bit plane." A bit in the plane is "set"that is, logical 1 -if the signal from the corresponding pixel falls within the threshold, otherwide the bit is "cleared"-that is, logical 0 . To identify the boundary of an object those bits that are set but have at least one adjacent bit cleared are identified. A significant microscopic object is then defined as one or several contiguous set bits completely surrounded by cleared bits.

Erosion is a process that can be repeated several times on the computerised image of a microscopical object. At each erosion all the bits that correspond with the boundary points of an object are cleared thereby creating a new boundary within the original one. The effect in terms of the microscopical object-for example, a single stained cell-is to

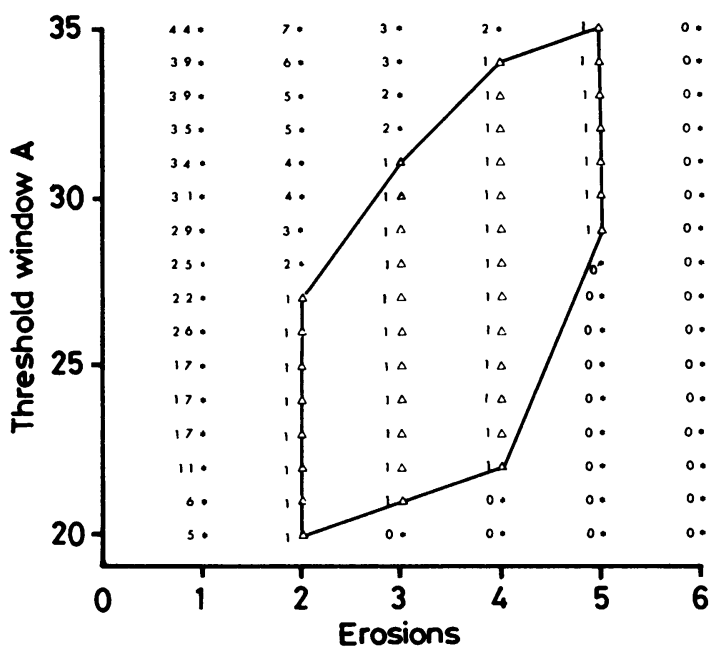

Fig. 2 Results of scanning a field containing one abnormal cell among normal cells. Entries show number of cells counted for different settings of parameters for threshold and erosion. Regions containing correct answer are outlined and settings marked as $\triangle$. Incorrect answers are shown as *.

reduce the size of the image of an object in the bit plane by the equivalent of twice the interpixel distance. As there are 256 pixels across and 256 down the field of the camera the interpixel distance is $1 / 255$ of the width of field viewed by the camera. In our system the distance in the microscope corresponding to the interpixel distance was $1.3 \mu \mathrm{m}$, and so each erosion reduced the diameter of the object as seen by the computer by $2.6 \mu \mathrm{m}$. Fig. 2 shows the effect of jointly varying the threshold and number of erosions for a test slide containing one dyskaryotic cell and about 50 normal squamous cells and polymorphs.

There is a certain elliptical area in which the single dysplastic cell is recognised and other objects in the field are ignored. Specificity increased with a decrease in the threshold (equivalent to an increase in threshold optical density) and an increase in the number or erosions up to a point at which the dysplastic cell itself was no longer recognised. Within the region in which identification was correct it was possible to confirm that the dysplastic cell was specifically recorded, as the monitor screen display that accompanies the process of erosion highlights this cell alone.

In subsequent studies an operating point was chosen from the centre of this region-that is, with the threshold set at 26 and with three erosions. Before embarking on a detailed analysis of the clinical material about 30 or 40 fields from different smear preparations were examined in an interactive manner to confirm that the chosen operating conditions did, in 
fact, give a reliable evaluation of the number of abnormal cells in a variety of materials. It was found that the number of adjacent cells within clusters could also be correctly evaluated provided that the cells did not overlap each other (Fig. 3b).

The area of field at this magnification was 0.11 $\mathrm{mm}^{2}$ and contained about 50 cells. For each smear a total of 10 independent fields were evaluated equivalent to $1 / 700$ of the total smear area and containing about 500 cells in total.

\section{Results}

On the basis of cytological findings the 37 cases examined were classified into five groups: apparently normal individuals (group 1), patients with cervical intraepithelial neoplasia (CIN/CIN I, CIN II, CIN III (groups 2, 3, 4)), and patients with invasive carcinoma of the cervix (group 5). Eighty seven monolayer preparations were prepared from smears from these patients. The important features of the monolayer preparations were the predominantly homogeneous distribution of the cells in the smear, an absence of overlapping, and a reduced background due to mucus and cellular debris (Fig. 3).

Table 1 shows the results of the examination of the smears by automated screening. The results were in agreement with the cytological findings, with the exception of two false negative and three false positive findings. In the slides stained by polyclonal antibodies the true positive counts ranged from one to 25 cells in 10 fields. There were three smears that gave a count of one and there were two counts of two cells, but the remaining counts ranged from four to 21 abnormal cells with a modal value of 12 cells.

As for the monoclonal antibodies, smears stained with these reagents generally did not show the same abnormal cell count as the corresponding slide stained with the polyclonal reagent. This feature was especially noticeable in the group with cervical intraepithelial neoplasia grade $\mathbf{I}$, in which there was one false negative result, four smears with only one abnormal cell counted, and the range of the remainder was between two and seven cells. The false nega-

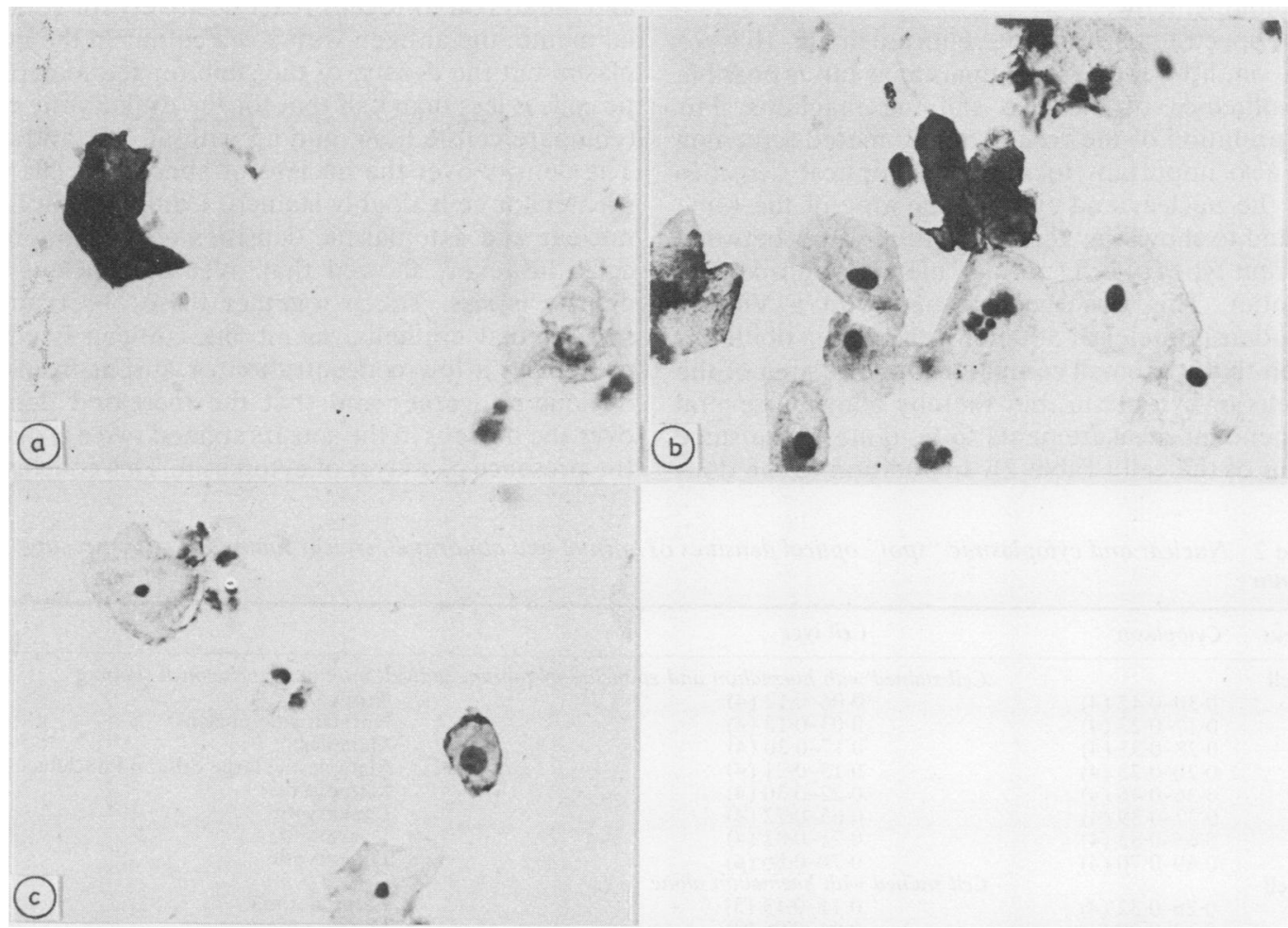

Fig. 3 (a) Part of cervical smear stained with haemalum and antiepithelial membrane antigen antibody and alkaline phosphatase coupled second antibody. Heavily stained irregularly shaped body is a dysplastic cell. $\times 450$. (b) Similar field stained with monoclonal antibody 8.33. Central cluster of abnormal cells was counted as three cells (automated system). $\times 300$. (c) Field containing metaplastic cell that stains weakly both by nuclear and immunocytochemical staining. $\times 190$. 
Table 1 Incidence of positive results from automated screening

\begin{tabular}{|c|c|c|c|c|c|}
\hline \multirow[t]{3}{*}{ Immunochemical reagent } & \multicolumn{5}{|c|}{ Diagnosis by Papanicolaou smear } \\
\hline & \multirow{2}{*}{$\begin{array}{l}\text { Normal } \\
(n=33)\end{array}$} & \multicolumn{3}{|c|}{ Cervical intraepithelial neoplasia } & \multirow{2}{*}{$\begin{array}{l}\text { Invasive } \\
\text { cancer } \\
(n=9)\end{array}$} \\
\hline & & $\begin{array}{l}\text { Grade I } \\
(n=19)\end{array}$ & $\begin{array}{l}\text { Grade II } \\
(n=9)\end{array}$ & $\begin{array}{l}\text { Grade III } \\
(n=17)\end{array}$ & \\
\hline $\begin{array}{l}\text { Polyclonal antibodies } \\
77.1 \\
8.30 .3\end{array}$ & $\begin{array}{l}3(n=19) \\
0(n=7) \\
0(n=7)\end{array}$ & $\begin{array}{l}8(n=8) \\
6(n=6) \\
4(n=5)\end{array}$ & $\begin{array}{l}4(n=4) \\
2(n=2) \\
3(n=3)\end{array}$ & $\begin{array}{l}9(n=9) \\
3(n=3) \\
4(n=5)\end{array}$ & $\begin{array}{l}3(n=3) \\
3(n=3) \\
3(n=3)\end{array}$ \\
\hline
\end{tabular}

A positive result was scored if at least one field examined showed a positive count of cells falling within the mask.

tive result found in the group with cervical intraepithelial neoplasia grade III, using the monoclonal antibody 8.30.3, may have been due to sampling error as the corresponding slide stained by the polyclonal reagent gave a count of two abnormal cells. The staining with the monoclonal reagent was weaker than with the polyclonal reagent. It was noticed that with the variables chosen for use with the polyclonal reagent some cells stained by the monoclonal reagent were missed and could only be included in the mask by increasing the width of the threshold window.

The spectra of absorption reported in Fig. $1 \mathrm{~b}$ were made on different cells to separate as far as possible the influence of the two staining reactions. For interpretation of the results of automated screening it is also important to determine optical densities over the nuclear and cytoplasmic area of the same cell and to show how this correlation varies between different types of cell (normal, metaplastic, and dyskaryotic). This was accomplished with a Vickers microdensitometer at $500 \mathrm{~nm}$ with an area of illumination that was small compared with the area of the nucleus or cytoplasm, but thereby allowing several independent measurements to be done in the same region of the cell (Table 2). In addition to the dou- bly stained material some measurements were made on smears stained with haemalum alone.

Normal cells show little difference between specimens singly or doubly stained as they do not stain for epithelial membrane antigen (compare cells $\mathrm{a} 1$ and $\mathrm{a} 2$ with b1). The larger nuclei of the abnormal cells may be less intensively stained with haemalum than the small nuclei of the normal cells (cells b1 and b5), but this is not found consistently (cell b4).

Both the occasionally stained metaplastic cells and the dyskaryotic cells react positively for epithelial membrane antigen with a red colour in the cytoplasm, but the density of the stain for the metaplastic cells is less than half that for the dyskaryotic cells (compare cells a3, a4, and a5 with a6, a7, and a8). The density over the nucleus of abnormal cells was greater for cells doubly stained. Comparison of the nuclear and cytoplasmic densities of the abnormal cells, however, showed that over the nucleus the density is less. Taken together these observations suggest that epithelial membrane antigen is either present in a low concentration or absent from the nucleus altogether and that the increased density over the nucleus in the smears stained twice is due to the presence of a layer of cytoplasm. This conclusion

Table 2 Nuclear and cytoplasmic "spot" optical densities of normal and abnormal cervical squamous cells measured at $500 \mathrm{~nm}$

\begin{tabular}{llcl}
\hline Nucleus & Cytoplasm & Cell type \\
\hline (a) cell & & Cell stained with haemalum and epithelial membrane antigen immunocytochemical staining & Normal \\
1 & $0.30-0.42(4)$ & $0.06-0.12(4)$ & Normal, pale nucleus \\
2 & $0.17-0.23(4)$ & $0.03-0.13(4)$ & Metaplastic \\
3 & $0.28-0.33(4)$ & $0.17-0.30(4)$ & Metaplastic, large cell, and nucleus \\
4 & $0.20-0.23(4)$ & $0.13-0.21(4)$ & Metaplastic \\
5 & $0.36-0.46(4)$ & $0.22-0.30(4)$ & Dyskaryotic \\
6 & $0.37-0.39(4)$ & $0.65-0.72(4)$ & Dyskaryotic \\
7 & $0.65-0.83(4)$ & $0.71-0.82(4)$ & Dyskaryotic \\
8 & $0.69-0.70(4)$ & $0.78-0.86(4)$ & \\
$(b)$ cell & & Cell stained with haemalum alone & Large normal \\
1 & $0.26-0.32(4)$ & $0.11-0.13(3)$ & Dyskaryotic \\
2 & $0.18-0.20(4)$ & $0.04-0.08(3)$ & Dyskaryotic \\
3 & $0.23-0.25(3)$ & $0.04-0.05(2)$ & Small dysplastic \\
4 & $0.41-0.46(3)$ & $0.18-0.20(4)$ & Abnormal \\
5 & $0.11-0.16(4)$ & $0.06(2)$ & \\
\hline
\end{tabular}

Numerical values show range of densities followed by number of observations in parentheses. Measuring site within cell were chosen to display both typical and extreme densities. 
is confirmed by observations in the spectrum of the nuclear region of a heavily stained cell. The maximum absorption was at $510 \mathrm{~nm}$, confirming a considerable contribution of cytoplasm.

\section{Discussion}

The main aim of this study was to test a new approach for the image analysis of cervical smears. Immunocytochemical staining as a basis for automated screening analyses has not previously been described. For this procedure we used combined cytoplasmic and nuclear optical density after immunocytochemical and nuclear staining. The abnormal cells were detected by their strongly positive expression of epithelial membrane antigen. The counter staining of the nuclei with haemalum has a complementary effect, which may increase the sensitivity of the method and allow the discrimination of individual cells in small groups. Immunocytochemical staining of cervical smears, produces an occasional staining of certain metaplastic cells, which can be problematic. ${ }^{10}$ Both immunocytochemical and nuclear staining were weaker with metaplastic than with dysplastic cells (Fig. 3c). Staining for both enhances the discrimination. Those metaplastic cells encountered in some of the smears from this study were not counted as abnormal by inclusion in the mask.

The process of erosion and also the setting of thresholds of optical density both play a part in determining overall specificity of the system. According to the evidence of Fig. 2, at least two erosions are required before the background from normal squamous cells, polymorphs, and debris is eliminated. Two erosions eliminate all objects with minimum diameter of above threshold staining of less than $5.2 \mu \mathrm{m}$. If the interior is not optically dense, however, erosions will occur both at an interior and at an exterior boundary; the overall effect will be twice as great, so that with two erosions objects less than $10.4 \mu \mathrm{m}$ will be lost.

Although the nuclear staining viewed at $500 \mathrm{~nm}$ is weak in comparison with the cytoplasmic staining (Table 2), the absence of a nuclear stain would create a weakly stained centre of the cell, which could suffer excessive erosion. Only with dyskaryotic cells does there seem to be a large area of heavy staining affecting both the nucleus and the cytoplasm, and the nuclear area includes a considerable cytoplasmic contribution to the optical density.

A comparison of findings on image analysis with the cytological findings showed differences in five out of 87 . In the three positive preparations there were problems with the preparation of the slides that are unlikely to occur as often in an automated laboratory-namely, the inclusion in the mounted slides of air bubbles or overlapping clumps of cells. One false negative result was obtained in a specimen with mild dyskaryosis with very weak staining of the dyskaryotic cells when stained with the monoclonal antibody 8.30.3, and the other was in the group with cervical intraepithelial neoplasia grade III, in which occasional low counts were found by the polyclonal antibody. The rate of false positive error of $3 \%$ and the rate of false negative error of about $2 \%$ relative to the total number of specimens examined is encouraging for the further development of automated screening, in view of the sensitivity of the system to early lesions. Manual prescreening of good quality smears of difficult specimens has been reported to give rates of $4 \%$ (false positive) and $6 \%$ (false negative). ${ }^{16}$

In practice, specificity is of great importance if the need for human intervention in the automated process, with a consequent of both time and resources, is to be avoided. The rate of scanning is also important, however, as different procedures can vary considerably, depending on the variable to be evaluated and the computer power available. ${ }^{7-9}$ In this preliminary study a total of 500 cells ( 10 fields) were scanned, a number that has been regarded as suitable for effective diagnosis. ${ }^{41}$ The occasional observation of a single abnormal cell in 10 fields, however, suggests that a better result would be obtained by at least doubling the number of fields examined, so as to reduce the chance of a false negative result by statistical fluctuations.

To scan 20 fields would require one minute with the system as we have used it. The TAS system uses basic computer operations common to a variety of systems for recognising patterns. The process that seems to limit the rate of evaluation is the time required for the repeated erosions. An increase in the rate of processing of the samples would be expected if the magnification between smear and videoscan was reduced. This would increase the number of cells in each field and reduce the number of erosions necessary for a given reduction of the dimensions in the computerised image. This increase, must be combined with three periods of incubation in the immunoenzymatic procedure, which require three hours plus time for intermediate washes. About 200 smears would need to be in preparation at any one time in order to match an evaluation time of one minute. Preliminary work suggests that the time for incubation could be reduced without a reduction in the quality of staining.

This study shows that immunocytochemistry may enhance the discriminating power of automated screening of cervical smears. More work is needed to test the reproducibility, stability, and standardisa- 
tion of the staining procedures. The use of monoclonal antibodies could help to make the procedure more reproducible in the long term, though our results suggest that staining with these antibodies would benefit from using a mixture of antibodies with several independent epitopes. Any procedure that enhances the contrast between normal and abnormal cells is likely to reduce the cost of the equipment necessary for analysis of the image. On the basis of the present study automated screening of immunocytochemically stained cervical smears is considered to be worthy of further study as a potentially speedy and reliable method.

We thank Dr DR Cheetham of the Praed Street special clinic, Dr MC Anderson of the Western Samaritan Hospital for Women for providing the cervical material, and Dr D Coleman and her staff at the cytology unit, St Mary's Hospital, Paddington, for providing the cytological evaluation of some of the materials. Dr T Hughes and Mr J Wiczkowski of Leitz Instruments, Luton, Bedfordshire, provided advice on the use of the TAS instrument and its operating system.

We thank Dr MG Ormerod and Mrs K Steele, Institute of Cancer Research, for supplying the polyclonal antiserum to epithelial membrane antigen and Dr IC Summerhayes (ICR) for the monoclonal antibodies. Dr N Loveridge, Dr J Chayen and Dr L Bitensky of the Kennedy Institute for Rheumatology, Hammersmith, kindly measured the absorption spectra and optical densities. Additional microdensitometric measurements were made at the department of biochemical pathology, University College, London, by Professor PA Riley and Dr DJ Spargo.

We thank Professor KA Porter, department of experimental pathology, St Mary's Hospital, Paddington, and Dr RJ Coffey, University College Hospital, for reading the manuscript. Professor AM Neville kindly supported the work.

\section{References}

' Erhardt R, Reinhardt ER, Schlipf W, Bloss WH. A system for fast automated cell segmentation, cell image analysis and feature extraction based on TV-image pickup and parallel processing. Anal Quant Cytol 1980;2:25-40.

${ }^{2}$ Koss LA, Bartels PH, Bibbo M, Freed SZ, Taylor J, Wied GL.
Computer discrimination between benign and malignant urothelial cells. Acta Cytol (Baltimore) 1975;19:378-91.

${ }^{3}$ Spriggs AI, Diamond RA, Meyer EW. Automated screening for cervical smears. Lancet 1968 ; i:359-60.

${ }^{4}$ Tolles WE, Horvarth WJ, Bostrom RC. Study of the quantitative characteristics of exfoliated cells from the female genital tract. I. Measurement methods and results. Cancer 1961;14:454-73.

5 Tolles WE, Horvarth WJ, Bostrom RC. A study of the quantitative characteristics of exfoliated cells from the female genital tract. II. Suitability of quantitative cytological measurements for automated pre-screening. Cancer 1961;14:455-68.

- Ploem-Zaaijer JJ, Beyer-Boon ME, Leyte-Veldstra L, Ploem JS. Cytofluorometric and cytophotometric DNA measurements of cervical smears stained using a new bi-color method. In: Pressman NJ, Wied GL, eds. The automation of cancer cytology and cell image analysis. Chicago: Tutorials of Chicago, 1979:225-35.

${ }^{7}$ Husain OAN, Skinner GK, Tatham PER, Tucker JH. Highlighting of progress by research groups in cytology automation: United Kingdom. In: Pressman NJ, Wied GL, eds. The automation of cancer cytology and cell image analysis. Chicago: Tutorials of Chicago, 1979:89-93

${ }^{*}$ Read JS. Borovec RT, Bartels PH, et al. A fast image processor for locating cell nuclei in uterine specimens. In: Pressman NJ, Wied GL, eds. The automation of cancer cytology and cell image analysis. Chicago: Tutorials of Chicago, 1979:143-55

' Mukawa A, Tanaka N, Ikeda H, Ueno T. A practical evaluation of the CYBEST system in the use of mass-screening on epidermoid carcinoma of the uterine cervix. In: Pressman NJ, Wied GL, eds. The automation of cancer cytology and cell image analysis. Chicago: Tutorials of Chicago, 1979:193-9

${ }^{10}$ Valkova B, Ormerod MG, Moncrieff D, Coleman DV. Epithelial membrane antigen in cells from the uterine cerviximmunocytochemical staining of cervical smears.J Clin Pathol 1984;37:984-9.

" Schwarz G, Schwarz M, Schenck U. Effect of the special properties of monolayer cell preparations for automated cervical cytology on visual evaluation and classification with an estimation of the number of cells required to be screened. Anal Quant Cytol 1983;5: 189-93.

${ }^{12}$ To A, Dearnaley DP, Ormerod MG, Ganti G, Coleman DV. Indirect immunoalkaline phosphatase staining of cytologic smears of serou effusions for tumour marker studies. Acta Cytol 1983;27:109-13.

${ }^{13}$ Bancroft JD. Histochemical techniques, 2nd ed. London: Butterworths, 1975:243.

${ }^{14}$ Drury RAB, Wallington EA. Carleton's histological technique, 5th ed. London: Oxford University Press, 1980.

is Baker JR. Experiments on the action of mordants. 2. Aluminium-haematein. Quarterly Journal of Microscopical Sciences 1962;103:493-517.

${ }^{16}$ Collins DN, Kaufman W. Quality evaluation of cytology laboratories in New York state: Expanded program 1971-73. Acta Cytol 1974;18:404-13.

Requests for reprints to: Dr DJR Laurence, Ludwig Institute for Cancer Research (London Branch), Royal Marsden Hospital, Clifton Avenue, Sutton, Surrey SM2 5PX, England. 